\title{
Discovery of eleven new ZZ Ceti stars ${ }^{\star}$
}

\author{
B. G. Castanheira ${ }^{1,2}$, S. O. Kepler ${ }^{1}$, F. Mullally ${ }^{2}$, D. E. Winget ${ }^{2}$, D. Koester ${ }^{3}$, B. Voss $^{3}$, \\ S. J. Kleinman ${ }^{4}$, A. Nitta ${ }^{4}$, D. J. Eisenstein ${ }^{5}$, R. Napiwotzki ${ }^{6}$, and D. Reimers ${ }^{7}$
}

\footnotetext{
${ }^{1}$ Instituto de Física, Universidade Federal do Rio Grande do Sul, 91501-900 Porto-Alegre, RS, Brazil e-mail: barbara@if.ufrgs.br

2 Department of Astronomy and McDonald Observatory, University of Texas, Austin, TX 78712, USA

3 Institut für Theoretische Physik und Astrophysik, Universität Kiel, 24098 Kiel, Germany

4 Sloan Digital Sky Survey, Apache Pt. Observatory, PO Box 59, Sunspot, NM 88349, USA

5 Steward Observatory, University of Arizona 933 N. Cherry Ave. Tucson, AZ 85721, USA

${ }^{6}$ Centre for Astrophysics Research, University of Hertfordshire, College Lane, Hatfield AL10 9AB, UK

7 Hamburger Sternwarte, Gojensbergweg 112, 21029 Hamburg, Germany
}

Received 24 May 2005 / Accepted 1 December 2005

\section{ABSTRACT}

We report the discovery of eleven new ZZ Cetis using telescopes at OPD (Observatório do Pico dos Dias/LNA) in Brazil, the 4.1 m SOAR (Southern Astrophysical Research) telescope at Cerro Pachon, Chile, and the $2.1 \mathrm{~m}$ Otto Struve telescope at McDonald observatory. The candidates were selected from the SDSS (Sloan Digital Sky Survey) and SPY (ESO SN Ia progenitor survey), based on their $T_{\text {eff }}$ obtained from optical spectra fitting. This selection criterion yields the highest success rate of detecting new ZZ Cetis, above $90 \%$ in the $T_{\text {eff }}$ range from 12000 to $11000 \mathrm{~K}$. We also report on a DA not observed to vary, with a $T_{\text {eff }}$ placing the star close to the blue edge of the instability strip. Among our new pulsators, one is slightly cooler than this star for which pulsations were not detected. Our observations are an important constraint on the location of the blue edge of the ZZ Ceti instability strip.

Key words. stars: white dwarfs - stars: variables: general - stars: oscillations

\section{Introduction}

White dwarf stars are the end point of the evolution of $95-98 \%$ of all stars. As they cool, white dwarf stars pass through three distinct instability strips, depending on their temperatures, atmospheric composition, and the element that drives pulsation: carbon and/or oxygen in DOVs, helium in DBVs, or hydrogen in DAVs or ZZ Cetis. Observationally, the DA instability strip ranges in effective temperature ( $\left.T_{\text {eff }}\right)$ from 12270 to $10850 \mathrm{~K}$ (Bergeron et al. 2004; Mukadam et al. 2004; and Gianninas et al. 2005). While Bergeron's instability strip is pure, Mukadam et al. (2004) and Mullally et al. (2005) did not detect light variability in a significant number of stars located within the $T_{\text {eff }}$ and $\log g$ boundaries of the instability strip. If the instability strip is contaminated (occupied by non-pulsators of the same spectral type as pulsators), pulsation would no longer be a normal stage of white dwarf evolution. However, because of destructive interference between modes, some white dwarf stars can appear to be constant for timescales of 3-4 h. Another important issue is that some known pulsating white dwarf stars show amplitudes as low as 4 mma (e.g. Kanaan et al. 2005), so we have to reach at least this limit to determine whether a star pulsates or not; many stars not observed to vary by Mukadam et al. (2004) have detection limits above 4 mma.

Pulsations are global; each periodicity is an independent measurement of the interior. This gives rise to asteroseismology: the study of stars below the photosphere through measurement of their pulsations. Comparing the observed pulsation spectrum to models of the stellar interior through asteroseismology allows for stellar mass determination (Winget et al. 1990; Bradley \& Winget 1994) and even constrains the value of the $\mathrm{C}^{12}(\alpha, \gamma) \mathrm{O}^{16}$ cross section (e.g. Metcalfe et al. 2002), which can only be measured in a terrestrial laboratory at energy levels eight orders of magnitude higher. The cooling time scales of DAVs (Kepler et al. 2000, 2005; Mukadam et al. 2003) can be used to calibrate the age of the galactic morphological components (Winget et al. 1987; Hansen et al. 2002; von Hippel 2005) by observing field stars, open and globular clusters up to magnitudes to include the turnoff of the white dwarf cooling sequence.

Pulsating white dwarf stars are also important for the study of extreme physics: internal crystallization (Winget et al. 1997; Kanaan et al. 2005), neutrino cooling (Kawaler et al. 1986; Winget et al. 2004) and axion emission (Córsico et al. 2001; Kepler 2004; Kim et al. 2005). Both particles can be created at 
temperatures and densities found in white dwarf cores and consequently must be taken into account in the cooling models.

Quasar surveys, like SDSS (Sloan Digital Sky Survey), HE (Hamburg ESO) and 2dF (Two Degree Field), are increasing the number of spectroscopically identified white dwarf stars; quasars and white dwarf stars have similar colors. As these new white dwarf stars are fainter than the ones previously known, but with similar $T_{\text {eff }}$, we are able to study a sample of stars at larger distances, with a wider range of progenitor metallicity. The study of their chemical composition is of interest if we are to use SN Ia as standard candles, that are products of mass accretion onto white dwarf stars.

Mukadam et al. (2004) show that at least $90 \%$ of the SDSS candidates with $12000 \geq T_{\text {eff }} \geq 11000 \mathrm{~K}$ are pulsators. We selected our targets based on the $T_{\text {eff }}$ we derive by fitting the whole optical spectra, as described by Kleinman et al. (2004). For the candidates with spectra obtained by the SPY survey (e.g. Napiwotzki et al. 2003), we derive $T_{\text {eff }}$ by fitting only the hydrogen line profiles. In both fitting procedures, we use the same model atmosphere grid, developed by Detlev Koester (similar to that described in Finley et al. 1997). We only obtained time series photometry of stars with $T_{\text {eff }}$ inside or close to the ZZ Ceti instability strip. All observed stars, but one, turned out to be pulsators.

In this paper, we report the discovery of eleven new pulsators and one star not observed to vary down to a detection limit of $3.75 \mathrm{mma}$.

\section{Observations}

We observed some of our candidates at Observatório Pico dos Dias, LNA, in Brazil. For these observations, we used the 1.6-m telescope, with a frame transfer CCD 301, focal reducer, and no filters. The CCD has a quantum efficiency of $60 \%$ at around $4000 \AA$, the prefered wavelength range to observe ZZ Cetis to maximize the pulsation amplitude versus CCD efficiency. The integration times range from 15 to $45 \mathrm{~s}$, depending on readout times, weather conditions, and apparent stellar magnitude. We observed without filters because the g-mode pulsations in ZZ Ceti stars are coherent at all optical wavelengths (Robinson et al. 1982). We also observed bright candidates with the 0.6-m Zeiss telescope. We used the CCD 106, which has a $7.5 \mathrm{~s}$ readout, and integrations of 30 to $60 \mathrm{~s}$.

In June and July 2005, we used the SOAR Optical Imager, a mosaic of two EEV $2048 \times 4096$ CCDs, thinned and back illuminated, with an efficiency around $73 \%$ at $4000 \AA$, at the cassegrain focus of the 4.1-m SOAR telescope. We observed in fast readout mode, with the CCDs binned $4 \times 4$, to decrease the readout+write time to $6.4 \mathrm{~s}$, and still achieve a $0.354^{\prime \prime} /$ pixel resolution. The exposure times ranged from 20 to $40 \mathrm{~s}$. All observations were obtained with a Johnson B filter.

We also observed with the Otto Struve 2.1-m telescope at McDonald Observatory, using the Argos camera, a frame transfer CCD in the prime focus, with a BG40 filter to reduce the scatter from sky (Nather \& Mukadam 2004). The integration times were 20 and $30 \mathrm{~s}$.

In Table 1, we show the journal of observations for all stars.
The technique used to detect variability is differential time series photometry, comparing the targets with the other stars in the same field, to minimize the effects of sky and transparency fluctuations.

For each run, we extracted light curves using the IRAF script hsp, developed by Antonio Kanaan, using different aperture sizes and select the light curve with either the lowest noise level or the highest signal-to-noise rate (SNR) in the Fourier transform.

\section{Results}

In Table 2, we list the new variable stars and their physical parameters derived from optical spectra, using an improved model grid similar to that described in Finley et al. (1997). We fit the candidates from SDSS using the whole optical spectra and photometry, as in Kleinman et al. (2004), while for the candidate from HE we used only the hydrogen lines in the optical spectra, because it is independent of the flux calibration and the SNR is high enough.

In Figs. 1 and 2, we show the light curves on the left panels and the Fourier transform (FT) on the right. We show only a small part of the light curves, but the FTs were calculated for the entire dataset for each target. The lower detection limit in frequency is $2 / T$, where $T$ is the total length of the light curve, while the higher is the Nyquist frequency, $f_{\text {Nyquist }}=2 / t_{\text {exp }}$.

The criterion we used to determine which peaks are real in the discrete FT is to adopt an amplitude limit such that the probability of a noise peak exceeding this value is only $1 / 1000$ (false alarm probability). We then repeatedly subtracted the largest signal in the FT until there were no more peaks above the detection limit (see Table 3). The uncertainties in frequency are, on average, $123 \mu \mathrm{Hz}$, equivalent to 1 s at $P \sim 100 \mathrm{~s}$.

Combining these eleven new ZZ Cetis with the previously known pulsators, we see a well defined instability strip in Fig. 3. The triangles represent previously discovered candidates from SDSS, the circles are the stars fitted by Bergeron et al. (2004), and the squares are from this paper. This is not a homogeneous sample, as the previous determinations of $T_{\text {eff }}$ and $\log g$ were not obtained with the same model grid (e.g. Bergeron et al. 2004; Koester \& Allard 2000), but the instability strip is restricted to a narrow range of temperature $12270 \mathrm{~K} \geq T_{\text {eff }} \geq 10850 \mathrm{~K}$. The pulsating star J235040.72-005430.9 (Mukadam et al. 2004) is clearly outside the ZZ Ceti instability strip. Despite this star being cooler than the red edge of the instability strip, its detected periods indicate it is a hot DAV. It is necessary to obtain a higher signal-to-noise spectrum, because the SDSS spectrum has $S N R \sim 12$, and analyze it with the line profile technique for an accurate determination of both $T_{\text {eff }}$ and $\log g$. Finally, it is necessary to increase the sample of variables and non-variables at the borders of the instability strip, to determine its exact boundaries.

The star J214723.7-001358.4 did not show variability during 2 independent runs, down to a limit of $3.75 \mathrm{mma}$. According to the SDSS spectrum, this star has $T_{\mathrm{eff}}=$ $12000 \pm 280$ and $\log g=7.92 \pm 0.11$, slightly hotter than the hottest ZZ Ceti from SDSS, reported in this paper, J215354.1-074330.7 (see Table 2 for details). 
Table 1. Journal of observations with the 1.6-m and 0.6-m telescope at OPD, the 4.1-m SOAR telescope, and the 2.1-m telescope at McDonald observatory. $\Delta T$ is the length of the observing run and $t_{\exp }$ is the integration time of each exposure.

\begin{tabular}{|c|c|c|c|c|c|}
\hline Star & Run start (UT) & $t_{\exp }(\mathrm{s})$ & $\Delta T(\mathrm{~h})$ & \# points & Telescope \\
\hline \multirow[t]{5}{*}{ HE 0031-5525 } & 2004-08-14 04:03 & 40 & 0.71 & 6464 & $1.6 \mathrm{~m}$ \\
\hline & 2004-08-18 05:09 & 40 & 3.53 & 253 & $0.6 \mathrm{~m}$ \\
\hline & 2004-08-19 04:00 & 30 & 4.80 & 418 & $0.6 \mathrm{~m}$ \\
\hline & 2004-09-10 03:59 & 20 & 2.34 & 422 & $1.6 \mathrm{~m}$ \\
\hline & 2004-09-11 02:31 & 30 & 0.88 & 105 & $1.6 \mathrm{~m}$ \\
\hline \multirow[t]{4}{*}{ SDSS J024922.3-010006.7 } & 2004-09-11 04:35 & 40 & 3.66 & 329 & $1.6 \mathrm{~m}$ \\
\hline & 2004-11-13 01:56 & 20 & 2.44 & 439 & $1.6 \mathrm{~m}$ \\
\hline & 2004-11-14 03:16 & 30 & 3.52 & 422 & $1.6 \mathrm{~m}$ \\
\hline & 2004-11-15 00:30 & 15 & 2.56 & 615 & $1.6 \mathrm{~m}$ \\
\hline \multirow{2}{*}{ SDSS J125710.5+012422.9 } & 2005-06-05 00:32 & 20 & 1.70 & 234 & $4.1 \mathrm{~m}$ \\
\hline & 2005-06-07 00:03 & 30 & 2.00 & 193 & $4.1 \mathrm{~m}$ \\
\hline \multirow[t]{2}{*}{ SDSS J153332.9-020600.0 } & 2004-08-10 22:06 & 20 & 1.95 & 351 & $1.6 \mathrm{~m}$ \\
\hline & 2004-08-11 21:56 & 20 & 1.81 & 325 & $1.6 \mathrm{~m}$ \\
\hline \multirow[t]{2}{*}{ SDSS J161837.2-002302.7 } & 2005-06-06 02:59 & 40 & 4.13 & 321 & $4.1 \mathrm{~m}$ \\
\hline & 2005-06-07 05:54 & 40 & 2.00 & 160 & $4.1 \mathrm{~m}$ \\
\hline \multirow[t]{3}{*}{ SDSS J164115.5+352140.6 } & 2005-06-06 08:15 & 20 & 2.42 & 436 & $2.1 \mathrm{~m}$ \\
\hline & 2005-06-09 07:14 & 30 & 0.40 & 48 & $2.1 \mathrm{~m}$ \\
\hline & 2005-06-09 09:35 & 30 & 1.15 & 138 & $2.1 \mathrm{~m}$ \\
\hline \multirow[t]{2}{*}{ SDSS J212808.4-000750.8 } & 2004-08-11 00:20 & 30 & 2.15 & 258 & $1.6 \mathrm{~m}$ \\
\hline & 2004-08-12 01:33 & 30 & 0.74 & 89 & $1.6 \mathrm{~m}$ \\
\hline \multirow[t]{2}{*}{ SDSS J213530.3-074330.7 } & 2004-08-11 02:43 & 30 & 1.71 & 205 & $1.6 \mathrm{~m}$ \\
\hline & 2004-08-12 02:20 & 30 & 1.06 & 127 & $1.6 \mathrm{~m}$ \\
\hline \multirow[t]{2}{*}{ SDSS J214723.7-001358.4 } & 2005-07-05 08:14 & 40 & 2.10 & 115 & $4.1 \mathrm{~m}$ \\
\hline & 2005-07-07 05:25 & 30 & 3.00 & 300 & $4.1 \mathrm{~m}$ \\
\hline \multirow[t]{2}{*}{ SDSS J215354.1-073121.9 } & 2005-06-06 07:33 & 30 & 2.00 & 200 & $4.1 \mathrm{~m}$ \\
\hline & 2005-06-07 08:36 & 30 & 2.00 & 200 & $4.1 \mathrm{~m}$ \\
\hline \multirow[t]{6}{*}{ SDSS J223135.7+134652.8 } & 2004-08-13 01:34 & 40 & 3.88 & 349 & $1.6 \mathrm{~m}$ \\
\hline & 2004-08-14 01:48 & 40 & 1.93 & 174 & $1.6 \mathrm{~m}$ \\
\hline & 2004-08-18 01:26 & 60 & 1.58 & 82 & $0.6 \mathrm{~m}$ \\
\hline & 2004-08-19 01:54 & 45 & 1.65 & 107 & $0.6 \mathrm{~m}$ \\
\hline & 2004-09-10 00:30 & 30 & 3.14 & 377 & $1.6 \mathrm{~m}$ \\
\hline & 2004-09-10 23:57 & 30 & 2.43 & 292 & $1.6 \mathrm{~m}$ \\
\hline SDSS J230726.6-084700.2 & 2004-08-11 04:32 & 40 & 1.28 & 115 & $1.6 \mathrm{~m}$ \\
\hline
\end{tabular}

Table 2. List of properties of the new variables. The $T_{\text {eff }}$ and $\log g$ were derived from optical spectra, with the same model grid, despite different fitting procedures for HE and SDSS stars.

\begin{tabular}{cccccc}
\hline \hline Star & RA (2000) & Dec (2000) & $T_{\text {eff }}(\mathrm{K})$ & $\log g$ & $g(\mathrm{mag})$ \\
\hline HE 0031-5525 & $00: 33: 36$ & $-55: 08: 37$ & $11480 \pm 30$ & $7.65 \pm 0.02$ & 15.94 \\
SDSS J024922.3-010006.7 & $02: 49: 22.3$ & $-01: 00: 06.7$ & $11060 \pm 110$ & $8.31 \pm 0.10$ & 19.08 \\
SDSS J125710.5+012422.9 & $12: 57: 10.5$ & $+01: 24: 22.9$ & $11520 \pm 160$ & $8.36 \pm 0.09$ & 18.65 \\
SDSS J153332.9-020600.0 & $15: 33: 32.9$ & $-02: 06: 00.0$ & $11350 \pm 40$ & $8.20 \pm 0.02$ & 16.62 \\
SDSS J161837.2-002302.7 & $16: 18: 37.2$ & $-00: 23: 02.7$ & $10860 \pm 160$ & $8.16 \pm 0.12$ & 19.26 \\
SDSS J164115.5+352140.6 & $16: 41: 15.5$ & $+35: 21: 40.6$ & $11230 \pm 160$ & $8.43 \pm 0.10$ & 19.04 \\
SDSS J212808.4-000750.8 & $21: 28: 08.4$ & $-00: 07: 50.8$ & $11440 \pm 100$ & $8.29 \pm 0.07$ & 17.97 \\
SDSS J213530.3-074330.7 & $21: 35: 30.3$ & $-07: 43: 30.7$ & $11190 \pm 120$ & $7.67 \pm 0.09$ & 18.59 \\
SDSS J215354.1-073121.9 & $21: 53: 54.1$ & $-07: 31: 21.9$ & $11930 \pm 130$ & $8.07 \pm 0.06$ & 18.45 \\
SDSS J223135.7+134652.8 & $22: 31: 35.7$ & $+13: 46: 52.8$ & $11080 \pm 100$ & $7.95 \pm 0.07$ & 18.63 \\
SDSS J230726.6-084700.2 & $23: 07: 26.6$ & $-08: 47: 00.2$ & $11060 \pm 110$ & $8.19 \pm 0.09$ & 18.83 \\
\hline
\end{tabular}

However, the temperatures derived from SDSS optical spectra have external uncertainties larger than $300 \mathrm{~K}$, as demonstrated from their duplicate spectra; the uncertainties we quote in Table 2 are the internal uncertainties in the $\chi^{2}$ fitting, assuming no correlation between $T_{\text {eff }}$ and $\log g$. We conclude that our observations are still consistent with a pure instability strip, but it does not exclude possible contaminations, as the strip covers only $\sim 1200 \mathrm{~K}$. To solve this problem, we need spectra with $S N R \geq 50$ to achieve $\sigma_{T_{\text {eff }}} \leq 200 \mathrm{~K}$ and to re-observe the stars that do not show variability in the literature to decrease their detection limits below $4 \mathrm{mma}$. Meanwhile, the purity of the ZZ Ceti instability strip is still an open question.

Our results map not only the blue edge, but also the red edge of the instability strip. We discovered a pulsating star, 
Table 3. Periodicities identified in our data sets for the new ZZ Cetis, where $f$ is the frequency, $P$ is the period, and $A$ is the amplitude. The times of maxima $\left(T_{\max }\right)$ are given in relation to the $T_{0}$, in Barycentric Coordinate Time in days.

\begin{tabular}{|c|c|c|c|c|c|}
\hline Star & $f(\mu \mathrm{Hz})$ & $P(\mathrm{~s})$ & $A(\mathrm{mma})$ & $T_{\max }(\mathrm{s})$ & $T_{0}(\mathrm{BCT})$ \\
\hline \multirow[t]{3}{*}{ HE 0031-5525 } & 3035 & 329.5 & $2.5 \pm 1.0$ & $251 \pm 19$ & 2453231.6726750 \\
\hline & 3611 & 276.9 & $4.8 \pm 1.0$ & $245 \pm 13$ & \\
\hline & 3638 & 274.9 & $1.5 \pm 1.0$ & $40 \pm 41$ & \\
\hline \multirow[t]{2}{*}{ SDSS J024922.3-010006.7 } & 957 & 1045.2 & $10.9 \pm 1.5$ & $598 \pm 61$ & 2453259.6947745 \\
\hline & 994 & 1005.6 & $5.6 \pm 1.5$ & $254 \pm 115$ & \\
\hline SDSS J125710.5+012411.9 & 1104 & 905.8 & $46.7 \pm 5.7$ & $31 \pm 18$ & 2453526.525547 \\
\hline \multirow[t]{2}{*}{ SDSS J153332.9-020600.0 } & 3837 & 260.6 & $5.3 \pm 0.6$ & $130 \pm 14$ & 2453228.4215328 \\
\hline & 3879 & 257.8 & $4.3 \pm 0.6$ & $29 \pm 17$ & \\
\hline SDSS J161837.2-002302.7 & 1553 & 644.0 & $5.4 \pm 1.1$ & $238 \pm 22$ & 2453527.630436 \\
\hline SDSS J164115.5+352140.6 & 1276 & 809.3 & $27.3 \pm 4.6$ & $560 \pm 22$ & 2453527.750116 \\
\hline \multirow[t]{3}{*}{ SDSS J212808.4-000750.8 } & 3309 & 302.2 & $17.1 \pm 1.3$ & $52 \pm 4$ & 2453228.5198054 \\
\hline & 3638 & 274.9 & $11.0 \pm 1.3$ & $154 \pm 6$ & \\
\hline & 3461 & 289.0 & $9.7 \pm 1.4$ & $131 \pm 7$ & \\
\hline \multirow[t]{5}{*}{ SDSS J213530.3-074330.7 } & 1769 & 565.4 & $49.8 \pm 2.3$ & $12 \pm 5$ & 2453228.6189298 \\
\hline & 1958 & 510.6 & $16.8 \pm 2.2$ & $18 \pm 14$ & \\
\hline & 3094 & 323.2 & $13.0 \pm 2.3$ & $24 \pm 12$ & \\
\hline & 3334 & 299.9 & $22.9 \pm 2.3$ & $209 \pm 6$ & \\
\hline & 3549 & 281.8 & $13.3 \pm 2.3$ & $276 \pm 10$ & \\
\hline SDSS J215354.1-073121.9 & 4757 & 210.2 & $5.6 \pm 0.9$ & $15 \pm 5$ & 2453527.816768 \\
\hline \multirow[t]{5}{*}{ SDSS J223135.7+134652.8 } & 1413 & 707.5 & $17.1 \pm 1.7$ & $389 \pm 16$ & 2453230.5708122 \\
\hline & 1595 & 627.0 & $26.3 \pm 1.7$ & $172 \pm 10$ & \\
\hline & 1614 & 619.7 & $18.9 \pm 1.7$ & $104 \pm 13$ & \\
\hline & 1822 & 548.7 & $13.7 \pm 1.7$ & $264 \pm 16$ & \\
\hline & 2615 & 382.4 & $14.6 \pm 1.7$ & $369 \pm 10$ & \\
\hline \multirow[t]{2}{*}{ SDSS J230726.6-084700.2 } & 825 & 1212.2 & $25.6 \pm 2.4$ & $478 \pm 32$ & 2453228.6946388 \\
\hline & 1621 & 617.0 & $12.5 \pm 2.4$ & $457 \pm 33$ & \\
\hline
\end{tabular}

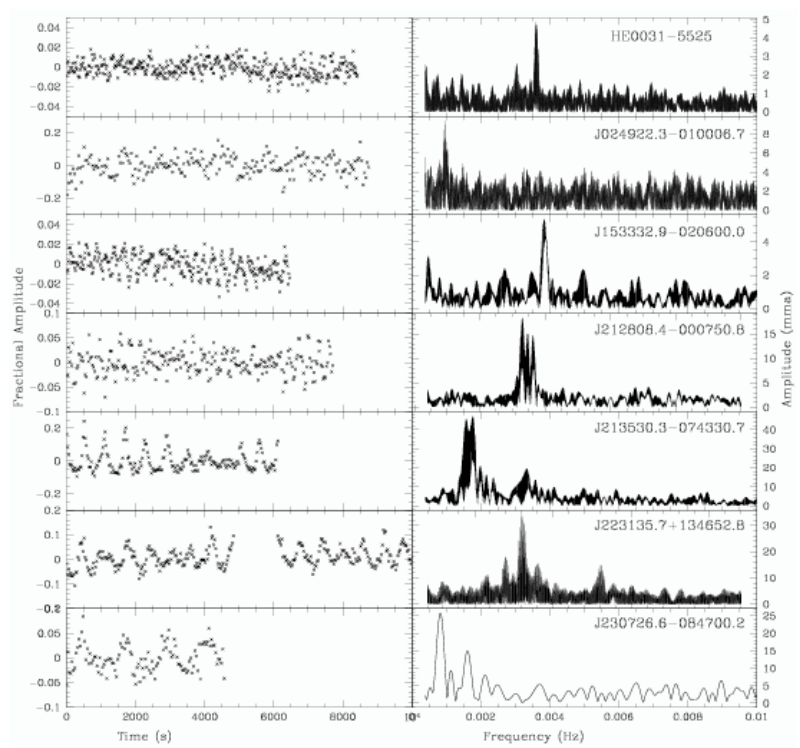

Fig. 1. The light curves of a single run (left panels) and the Fourier transforms of the complete data set (right panels) for the new ZZ Cetis, discovered at LNA.

J161837.2-002302.7, with $T_{\text {eff }}$ and periodicities characteristic of the red edge, even though the amplitudes are significantly lower than expected. This star will be extremely important as

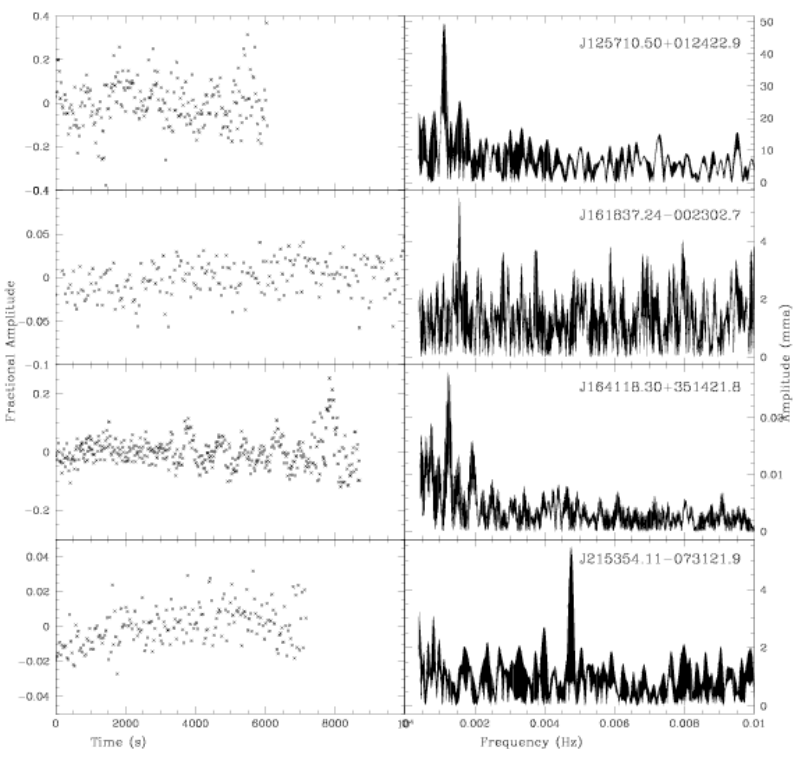

Fig. 2. The light curves of a single run (left panels) and the Fourier transforms of the complete data set (right panels) for the new ZZ Cetis, discovered at SOAR and McDonald observatory.

we try to understand how ZZ Cetis will stop pulsating around $T_{\text {eff }} \sim 10850 \mathrm{~K}$. 


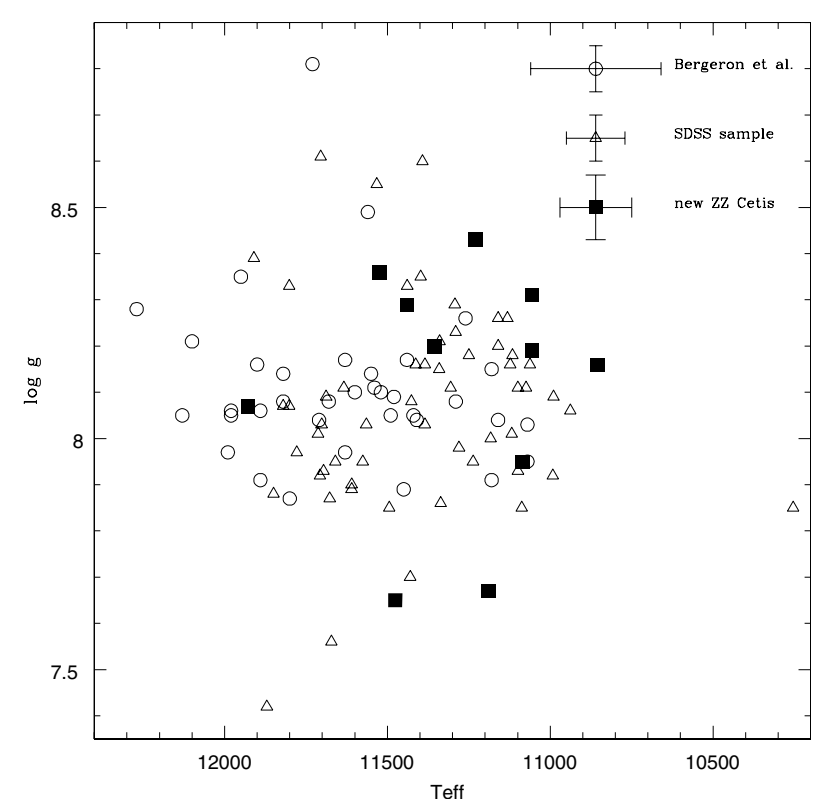

Fig. 3. The updated instability strip including all known ZZ Cetis. The circles are the stars described in Bergeron et al. (2004), the triangles are the pulsators discovered by Mukadam et al. (2004), Mullally et al. (2005), and Kepler et al. (2005b) from the SDSS, and the squares are the eleven new ZZ Cetis presented in this paper. The average uncertainties for each set are on the top right of the plot.

\section{Concluding remarks}

We report the discovery of eleven new pulsating DA stars, bringing the total to 106 known variables, all in the narrow temperature range, $12270 \mathrm{~K} \geq T_{\text {eff }} \geq 10850 \mathrm{~K}$, corresponding to the partial ionization of hydrogen and development of a sub-surface convection zone. Three stars, HE 0031-5525, SDSS J153332.9-020600.0 and SDSS J212808.4-000750.8, have pulsation characteristics of hot DAVs, even though their derived $T_{\text {eff }}$ are $11480 \mathrm{~K}, 11350 \mathrm{~K}, 11440 \mathrm{~K}$, respectively. However, $T_{\text {eff }}$ is not the only parameter that determines the position of the instability strip. Giovannini et al. (1998) demonstrate that the instability strip also depends on $\log g$. We therefore need to map the instability strip in both $T_{\text {eff }}$ and $\log g$ using accurate determinations of these parameters for the new variables.

Acknowledgements. Financial support: NASA origin grant, CAPES/UT grant, CNPq fellowship, D.K. and B.V. acknowledge support from the Deutsche Forschungsgemeinschaft (DFG, grant K0738/21-1).

\section{References}

Bergeron, P., Fontaine, G., Billères, M., Boudreault, S., \& Green, E. M. 2004, ApJ, 600, 404

Bradley, P. A., \& Winget, D. E. 1994, ApJ, 430, 850

Córsico, A. H., Althaus, L. G., Benvenuto, O. G., \& Serenelli, A. M. 2001, A\&A, 380, L17

Finley, D. S., Koester, D., \& Basri, G. 1997, ApJ, 488, 375

Gianninas, A., Bergeron, P., \& Fontaine, G. 2005, ApJ, 631, 1100

Giovannini, O., Kepler, S. O., Kanaan, A., et al. 1998, Baltic Astron., 7,131

Hansen, B. M. S., Brewer, J., Fahlman, G. G., et al. 2002, ApJ, 574, L155

Kanaan, A., Nitta, A., Winget, D. E., et al. 2005, A\&A, 432, 219

Kawaler, S. D., Winget, D. E., Iben, I., \& Hansen, C. J. 1986, ApJ, 302,530

Kepler, S. O., Mukadam, A., Winget, D. E., et al. 2000, ApJ, 534, L185

Kepler, S. O. 2004, Int. J. Mod. Phys. D, 13, 1493

Kepler, S. O., Costa, J. E. S., Castanheira, B. G., et al. 2005, ApJ, 634, 1311

Kepler, S. O., Castanheira, B. G., Saraiva, M. F. O., et al. 2005b, A\&A, 442,629

Kim, A., Winget, D. E., Montgomery, M. H., \& Sullivan, D. J. 2005, 14th European Workshop on White Dwarfs, ASP Conf. Ser., 334, 489

Kleinman, S. J., Harris, H. C., Eisenstein, D. J., et al. 2004, ApJ, 607, 426

Koester, D., \& Allard, N. F. 2000, Baltic Astronomy, 9, 119

Metcalfe, T. S., Salaris, M., \& Winget, D. E. 2002, ApJ, 573, 803

Mukadam, A. S., Kepler, S. O., Winget, D. E., et al. 2003, ApJ, 594, 961

Mukadam, A. S., Mullally, F., Nather, R. E., et al. 2004, ApJ, 607, 982

Mullally, F., Thompson, S. E., Castanheira, B. G., et al. 2005, ApJ, 625,966

Napiwotzki, R., Christlieb, N., Drechsel, H., et al. 2003, The Messenger, 112, 25

Nather, R. E., \& Mukadam, A. S. 2004, ApJ, 605, 846

Robinson, E. L., Kepler, S. O., \& Nather, R. E. 1982, ApJ, 259, 219

von Hippel, T. 2005, ApJ, 622, 565

Winget, D. E., Hansen, C. J., Liebert, J., et al. 1987, ApJ, 315, L77

Winget, D. E., Nather, R. E., Clemens, J. C., et al. 1990, ApJ, 357, 630

Winget, D. E., Kepler, S. O., Kanaan, A., Montgomery, M. H., \& Giovannini, O. 1997, ApJ, 487, L191

Winget, D. E., Sullivan, D. J., Metcalfe, T. S., Kawaler, S. D., \& Montgomery, M. H. 2004, ApJ, 602, L109 